\title{
Acknowledgement of reviewers for Volume 38
}

(C) Australasian College of Physical Scientists and Engineers in Medicine 2015

The journal is deeply indebted to the reviewers who generously donate their time and expertise to evaluate and improve manuscripts. The names of those reviewers

Shahab Anna Abdulla (Australia)

Trevor Ackerly (Australia)

Nahrizul Adib Kadri (Malaysia

Seyed Ali Aghayan (Iran)

Zohair Al-Ameen (Malaysia)

Trent Aland (Australia)

Bssam Al-Naami (Jordon)

Gokmen Altay (Turkey)

George Andrikopoulos (Greece)

Sankar Arumugam (Australia)

Andre Asena (Australia)

Supatana Auethavekiat (Thailand)

Phil Back (Australia)

Daniel Badger (Australia)

Ahamed Badusha (Ireland)

Seyyed Mehdi Bagherimofidi (Iran)

Mirza Baig (New Zealand)

Mariusz Bajger (Australia)

A. K. Bakshi (India)

Zoë Baldwin (Australia)

Dora Ballesteros (Colombia)

Wayne Beckham (Canada)

Hamid Behnam (Iran)

Yasmine Benchaib (Algeria)

Nicky Bertollo (Australia)

Toby Beveridge (Australia)

Madhava Bhat (Australia)

Giovanni Bibbo (Australia) who dealt with manuscripts submitted for consideration for Volume 38 are given below:

Katrina Biggerstaff (Australia)

B Bindhu (India)

Andrew Blair (New Zealand)

Samuel J. Blake (Australia)

Murk Bottema (Australia)

Zoe Brady (Australia)

Lorenzo Brualla (Germany)

Duncan Butler (Australia)

Martin Butson (Australia)

Andrew Campbell (Australia)

Martin Caon (Australia)

Martin Carolan (Australia)

Sha Chang (Australia)

Paul Charles (Australia)

Biswadeep Chaudhuri (India)

Pietro Cipresso (Italy)

John Costi (Australia

Benjamin Crouch (Australia)

Scott B. Crowe (Australia)

Jianfeng Cui (China)

Michael Oluwaseun Dada (Nigeria)

Mohammad Reza Daliri (Iran)

Marianne Schmid Daners (Switzerland)

Robert Davidson (Australia)

Yves DeDeene (Australia)

Kiran Dellimore (Netherlands)

Shane Dempsey (Australia)

Claire Dempsey (Australia) 
Larry DeWerd (USA)

Yan Ding (Australia)

Jian Dong (China)

Jason Dowling (Australia)

Francis Duck (United Kingdom)

Leon Dunn (Australia)

Efstathios Efstathopoulos (Greece)

Stefan Eichhorn (Germany)

Bilal Farouk El-Zayat (Germany)

Heiko Enderling (USA)

Shirin A. Enger (Canada)

Mahdad Esmaeili (Iran)

Maher Faik Esmaile (Malaysia)

Yubo Fan (China)

Sajjad Farashi (Iran)

Keith Faulkner (United Kingdom)

Andrew Fielding (Australia)

Shane Foley (Ireland)

Richard Fox (Australia)

Leonardo Franco (Spain)

Dan Fulea (Romania)

Romuald Gajewski (Australia)

Animesh Garg (USA)

Daniel Gasic (Denmark)

P. Geethanjali (India)

Mark Geurts (USA)

Ramon Gonzalez-Camarena (Mexico)

Miriam Gonzalez-Izal (Spain)

B. Gopinath (India)

Tina Gorjiara (Australia)

Atefeh Goshvarpour (Iran

Selvaraj Gunasunadri (India)

Amir Hakimi (Iran)

Cheol Han (Korea)

Christian Hansen (Denmark)

Valentina Hartwig (Italy)

William Kongto Hau (Hong Kong)

Annette Haworth (Australia)

Anna Hayton (Australia)

Mostafa Heydarian (Canada)

Kevin James Hickson (Australia)

Robin Hill (Australia)

Brendan Hill (Australia)

Monica Hinds (USA)

Lois Holloway (Australia)

Yigal S. Horowitz (Israel)

Andrew Howie (Australia)

Ming-Fa Hsieh (Taiwan)

Meng-Lun Hsueh (China)

Behzad Iravani (Iran)

Nima Jamshidi (Iran)

Suparerk Janjarasjitt (Thailand)

Munsif A Jatoi (Malaysia)
Peter Johnston (Australia)

Mattias Jonsson (Sweden)

Justin Joseph (India)

Noriyuki Kadoya (Japan)

Nahrizul Adib Kadri (Malaysia

Tanya Kairn (Australia)

Chandan Kumar Karmakar (Australia)

Ramgopal Kashyap (India)

Paul Keall (Australia)

Stephen Keevil (United Kingdom)

Yoshiaki Kiyanagi (Japan)

Konstantin Konstantinov (Austalia)

Tomas Kron (Australia)

S. S. Kumar (India)

Dae Cheol Kweon (Korea)

John Laban (New Zealand)

Alan Lai (Australia)

Marie-Véronique Le Lann (France)

GyuChang Lee (Korea)

Kam Lee (Australia)

Joerg Lehmann (Australia)

Peipeng Liang (China)

A. A. Linneger (USA)

Miao Liu (Korea)

Wei Liu (USA)

Zheng Feng Lu (USA)

Bernhard Ludewight (USA)

Suhuai Luo (Australia)

Jiasen Ma (USA)

Hamid Mahmoodian (Iran)

Nader Vahdani Manaf (Turkey)

Loredana G. Marcu (Romania)

Kazumi Masuda (Japan)

Fernando Mattioli (Brazil)

George McGill (Australia)

Donald McLean (Australia)

Donald McRobbie (Australia)

Peter Metcalfe (Australia)

Nigel Middlebrook (Australia)

M. Mohan (India)

Shinichiro Mori (Japan)

Yosry Morsi (Australia)

Iva Mrcela (Croatia)

Matthew Murray (Australia)

Ibrahim Mutlu (Turkey)

Ganesh R. Naik (Australia)

Christopher Njeh (USA)

Marta Parazzini (Italy)

Mike Partridge (United Kingdom)

Monica Patrascu (Romania)

Qiyu Peng (USA)

Peter Petersen (Denmark)

Angkoon Phinyomark (Canada) 
A Pichandi (India)

Tomasz Piotrowski (Poland)

Christopher Poole (Australia)

Mary Praveena (India)

Roger Price (Australia)

Yi Qian (Australia)

Alexandra Quinn (Ausralia)

Chitra Ragunath (Maldives)

Anna Ralston (Australia)

Suresh Rana (USA)

Neil Richmond (United Kingdom)

Howell Round (New Zealand)

Daniel Ruiz-Fernandez (Spain)

Sid Ahmed Sabeur (Algeria)

Mohammad Said Saidi (Iran)

Nong Sang (China)

Mark Schier (Australia)

Alison Scott (United Kingdom)

Ssang Hee Seo (Korea)

Gaurav Sethi (India)

John Shakeshaft (Australia)

Ajat Shatru Arora (India)

Lifeng Shen (China)

Seyed Alireza Mousavi Shirazi (Iran)

Almon Shiu (USA)

Paul Simpson (Australia)

Vivek Kumar Singh (India)

Ryan L. Smith (Australia)

D. Somasundaram (India)

Nathan Spreng (USA)

Natarajan Sriraam (India)

Christopher Stacey (United Kingdom)
Georg Stamm (Germany)

Zhonghua Sun (Australia)

Steven Sylvander (Australia)

Wei-Liang Tay (Singapore)

David Thiele (Australia)

Peter Thomas (Australia

Michael J. Thrippleton (United Kingdom)

Iuliana Toma-Dasu (Sweden)

Dharmendra Tripathi (India)

Gadyam Uthayakumar (India)

Anthony Venning (Australia)

Philip Vial (Australia)

Marko Vuskovic (USA)

Defeng Wang (Hong Kong)

Yinkun Wang (Canada)

George Warr (Australia)

Helen Warren-Forward (Australia)

David Waterhouse (Australia)

Luke Webb (Australia)

Anders Widmark (Norway)

Luke Wilkinson (Australia)

Dean Wilkinson (Australia)

Ivan Williams (Australia)

Tim Wilson (Canada)

Robert Woodward (Australia)

Shiqian Wu (China)

Wan Wu (USA)

Aitang Xing (Australia)

Sergei Zavgordni (Canada)

Sylwia Zawlodzka-Bednarz (Australia)

Benjamin Zwan (Australia) 